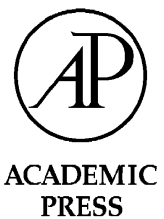

Available online at www.sciencedirect.com

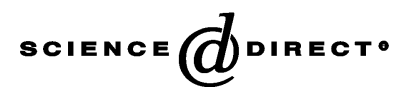

Journal of Sound and Vibration 267 (2003) 1047-1064

\title{
Interaction of eigenvalues in multi-parameter problems
}

\author{
A.P. Seyranian*, A.A. Mailybaev \\ Institute of Mechanics, Moscow State Lomonosov University, Michurinsky pr. 1, 119192 Moscow, Russia
}

Received 8 July 2002; accepted 29 October 2002

\begin{abstract}
The paper presents a new general theory of interaction of eigenvalues of matrix operators depending on parameters. Both complex and real eigenvalues are considered. Strong and weak interactions are distinguished, and their geometric interpretation on the complex plane is given. Mechanical examples are presented and discussed in detail.
\end{abstract}

(C) 2003 Elsevier Ltd. All rights reserved.

\section{Introduction}

Behaviour of eigenvalues with a change of parameters is a problem of general interest for applied mathematicians and natural scientists. This problem has many important applications in aerospace, mechanical, civil, and electrical engineering. Behaviour of eigenvalues depending on parameters is of a special value for vibration and stability problems, see, for example, the books by Bolotin [1], Panovko and Gubanova [2], Ziegler [3], Huseyin [4], Leipholz [5], Thompson [6], Thomsen [7], and Paidoussis [8].

The theory of interaction of eigenvalues, presented below, is based on the constructive perturbation methods, developed mostly by Vishik, Lyusternik, and Lidskii, see Refs. [9-11], and their extension to the multi-parameter case done by Seyranian [12,13], Mailybaev and Seyranian [14], and Seyranian and Kirillov [15]. In Refs. [12,13] the notion of strong and weak interactions of eigenvalues was introduced based on a Jordan form of the system matrix. If there are two eigenvectors corresponding to a double eigenvalue the interaction is called weak, and if there exists only one eigenvector with a double eigenvalue the interaction is called strong. It was shown that the strong interaction in one-parameter systems is characterized by two parabolae of equal curvature at the intersection point lying in perpendicular planes, while weak interaction occurs in

\footnotetext{
*Corresponding author. Tel.: + 7095-939-5478; fax: + 7095-939-0165.

E-mail address: seyran@imec.mus.ru (A.P. Seyranian).
} 

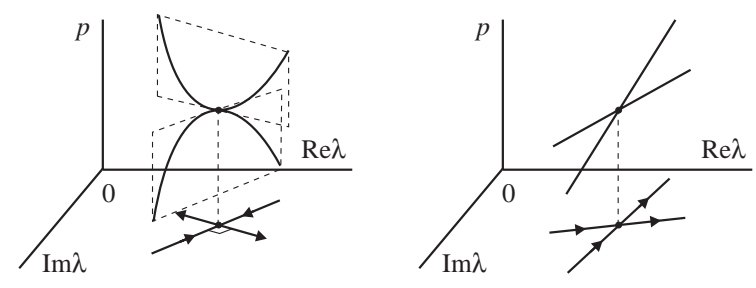

Fig. 1. Strong and weak interactions of eigenvalues in one-parameter problem.

the same plane; see Fig. 1. It is remarkable that the speed $\mathrm{d} \lambda / \mathrm{d} p$ of strong interaction tends to infinity at the intersection point, while weak interaction is characterized by finite interaction speed.

Some results on interaction of complex eigenvalues in vibrational systems were obtained by Seyranian [16], and Seyranian and Pedersen [17]. In these two papers the important mechanical effects like destabilization of a non-conservative system by infinitely small damping and transference of instability between eigenvalue branches were described and explained. Overlapping of frequency curves in circulatory systems was studied in Kirillov and Seyranian [18], and stability boundaries of Hamiltonian and gyroscopic systems were examined by Mailybaev and Seyranian [19] and Seyranian and Kliem [20].

In this paper, a general theory of interaction of two eigenvalues of matrix operators on the complex plane depending on multiple parameters is presented. Both complex and real eigenvalues are considered. Strong and weak interactions of eigenvalues, when one of the parameters is changed while increments of others remain constant, are distinguished based on a Jordan form of the system matrix. It is shown that the strong interaction of eigenvalues on the complex plane is described by hyperbolae with perpendicular asymptotes, if the double eigenvalue $\lambda_{0}$ is complex, and by a parabola and straight line, if $\lambda_{0}$ is real. Weak interaction of eigenvalues, characterized by two linearly independent eigenvectors at the point of coincidence, is studied. It is revealed that weak interaction is described by hyperbolae or a small elliptic bubble appearing from the point of the double eigenvalue $\lambda_{0}$ perpendicular to the plane of original interaction in the case of a real $\lambda_{0}$. And if $\lambda_{0}$ is complex the interacting eigenvalues keep or interchange their main directions of motion on the complex plane before and after the weak interaction. It is emphasized that the presented theory of interactions gives not only qualitative, but also quantitative results on behaviour of eigenvalues based only on the information at the initial point in the parameter space.

As mechanical examples, vibrational systems with coincident frequencies and stability of motion of a rigid panel in airflow are considered and discussed in detail.

\section{Strong interaction}

Consider an eigenvalue problem

$$
\mathbf{A u}=\lambda \mathbf{u},
$$

where $\mathbf{A}$ is a real $m \times m$ non-symmetric matrix with the elements $a_{i j}$ smoothly depending on a vector of real parameters $\mathbf{p}=\left(p_{1}, \ldots, p_{n}\right), \lambda$ is an eigenvalue, and $\mathbf{u}$ is a corresponding eigenvector. The eigenvalues $\lambda$ are determined from the characteristic equation $\operatorname{det}(\mathbf{A}-\lambda \mathbf{I})=0$, where $\mathbf{I}$ is the identity matrix. 
Assume that at $\mathbf{p}_{0}=\left(p_{1}^{0}, \ldots, p_{n}^{0}\right)$ the matrix $\mathbf{A}_{0}=\mathbf{A}\left(\mathbf{p}_{0}\right)$ possesses a double eigenvalue $\lambda_{0}$, as a root of the characteristic equation, with an eigenvector $\mathbf{u}_{0}$ and associated vector $\mathbf{u}_{1}$ satisfying the Jordan chain equations

$$
\begin{gathered}
\mathbf{A}_{0} \mathbf{u}_{0}=\lambda_{0} \mathbf{u}_{0}, \\
\mathbf{A}_{0} \mathbf{u}_{1}=\lambda_{0} \mathbf{u}_{1}+\mathbf{u}_{0} .
\end{gathered}
$$

Eqs. (2) mean that there is only one linearly independent eigenvector $\mathbf{u}_{0}$ corresponding to the double eigenvalue $\lambda_{0}$. Along with Eqs. (2), a left eigenvector $\mathbf{v}_{0}$ and associated vector $\mathbf{v}_{1}$ are defined by the left Jordan chain equations

$$
\begin{gathered}
\mathbf{v}_{0}^{\mathrm{T}} \mathbf{A}_{0}=\lambda_{0} \mathbf{v}_{0}^{\mathrm{T}}, \\
\mathbf{v}_{1}^{\mathrm{T}} \mathbf{A}_{0}=\lambda_{0} \mathbf{v}_{1}^{\mathrm{T}}+\mathbf{v}_{0}^{\mathrm{T}} .
\end{gathered}
$$

The eigenvectors and associated vectors of problems (2) and (3) are related by the equalities [9]

$$
\mathbf{v}_{0}^{\mathrm{T}} \mathbf{u}_{0}=0, \quad \mathbf{v}_{1}^{\mathrm{T}} \mathbf{u}_{0}=\mathbf{v}_{0}^{\mathrm{T}} \mathbf{u}_{1} \neq 0 .
$$

Note that the eigenvectors and associated vectors are not defined uniquely. Upon assuming that the vectors $\mathbf{u}_{0}$ and $\mathbf{u}_{1}$ are given, the following normalization conditions are introduced,

$$
\mathbf{v}_{0}^{\mathrm{T}} \mathbf{u}_{1}=1, \quad \mathbf{v}_{1}^{\mathrm{T}} \mathbf{u}_{1}=0,
$$

uniquely determining the vectors $\mathbf{v}_{0}$ and $\mathbf{v}_{1}$.

The aim of the paper is to study the behaviour of two eigenvalues $\lambda$, which are coincident and equal to $\lambda_{0}$ at $\mathbf{p}_{0}$, with a change of the vector of parameters $\mathbf{p}$ in the vicinity of the initial point $\mathbf{p}_{0}$. For this purpose, a variation $\mathbf{p}=\mathbf{p}_{0}+\varepsilon \mathbf{e}$ is assumed, where $\mathbf{e}=\left(e_{1}, \ldots, e_{n}\right)$ is a vector of variation with $\|\mathbf{e}\|=1$, and $\varepsilon>0$ is a small parameter. As a result, the matrix $\mathbf{A}$ takes the increment

$$
\mathbf{A}(\mathbf{p}+\varepsilon \mathbf{e})=\mathbf{A}_{0}+\varepsilon \mathbf{A}_{1}+\varepsilon^{2} \mathbf{A}_{2}+\cdots,
$$

where

$$
\mathbf{A}_{1}=\sum_{j=1}^{n} \frac{\partial \mathbf{A}}{\partial p_{j}} e_{j}, \quad \mathbf{A}_{2}=\frac{1}{2} \sum_{j, k=1}^{n} \frac{\partial^{2} \mathbf{A}}{\partial p_{j} \partial p_{k}} e_{j} e_{k},
$$

with the derivatives taken at $\mathbf{p}_{0}$. Due to these variations the eigenvalue $\lambda_{0}$ and the corresponding eigenvector $\mathbf{u}_{0}$ take increments, which can be given in the form of expansions [9]

$$
\begin{gathered}
\lambda=\lambda_{0}+\varepsilon^{1 / 2} \lambda_{1}+\varepsilon \lambda_{2}+\varepsilon^{3 / 2} \lambda_{3}+\cdots, \\
\mathbf{u}=\mathbf{u}_{0}+\varepsilon^{1 / 2} \mathbf{w}_{1}+\varepsilon \mathbf{w}_{2}+\varepsilon^{3 / 2} \mathbf{w}_{3}+\cdots .
\end{gathered}
$$

By substituting expressions (6) and (8) into eigenvalue problem (1), a chain of equations for the unknowns $\lambda_{1}, \lambda_{2}, \ldots$ and $\mathbf{w}_{1}, \mathbf{w}_{2}, \ldots$ is obtained. Solving these equations yields $[9,14]$

$$
\lambda_{1}= \pm \sqrt{\mathbf{v}_{0}^{\mathrm{T}} \mathbf{A}_{1} \mathbf{u}_{0}}, \quad \lambda_{2}=\left(\mathbf{v}_{0}^{\mathrm{T}} \mathbf{A}_{1} \mathbf{u}_{1}+\mathbf{v}_{1}^{\mathrm{T}} \mathbf{A}_{1} \mathbf{u}_{0}\right) / 2
$$

For the sake of convenience, the following notation is introduced

$$
a_{j}+\mathrm{i} b_{j}=\mathbf{v}_{0}^{\mathrm{T}} \frac{\partial \mathbf{A}}{\partial p_{j}} \mathbf{u}_{0}
$$




$$
\begin{gathered}
c_{j}+\mathrm{i} d_{j}=\frac{1}{2}\left(\mathbf{v}_{0}^{\mathrm{T}} \frac{\partial \mathbf{A}}{\partial p_{j}} \mathbf{u}_{1}+\mathbf{v}_{1}^{\mathrm{T}} \frac{\partial \mathbf{A}}{\partial p_{j}} \mathbf{u}_{0}\right), \\
\Delta p_{j}=p_{j}-p_{j}^{0}=\varepsilon e_{j}, \quad j=1,2, \ldots, n,
\end{gathered}
$$

where $a_{j}, b_{j}, c_{j}, d_{j}$ are real constants, and $\mathrm{i}$ is the imaginary unit. Then, using expressions (8)-(10) gives

$$
\lambda=\lambda_{0} \pm \sqrt{\sum_{j=1}^{n}\left(a_{j}+\mathrm{i} b_{j}\right) \Delta p_{j}}+\sum_{j=1}^{n}\left(c_{j}+\mathrm{i} d_{j}\right) \Delta p_{j}+o(\varepsilon) .
$$

Eq. (11) describes the increments of two eigenvalues $\lambda$, when the parameters $p_{1}, \ldots, p_{n}$ are changed under the assumption that $\varepsilon$ is small. Due to the condition $\|\mathbf{e}\|=1$ one has

$$
\|\Delta \mathbf{p}\|=\|\varepsilon \mathbf{e}\|=\varepsilon \ll 1 .
$$

Thus, inequality (12) implies that all the increments $\Delta p_{1}, \ldots, \Delta p_{n}$ are small for their absolute values.

From expression (11) it is seen that when only one parameter, say the parameter $p_{1}$, is changed while others remain unchanged $\Delta p_{j}=0, j=2, \ldots, n$, then the speed of interaction $\mathrm{d} \lambda / \mathrm{d} p_{1}$ is infinite at $p_{1}=p_{1}^{0}$. Indeed, following Eq. (11) yields

$$
\frac{\mathrm{d} \lambda}{\mathrm{d} p_{1}}= \pm \frac{1}{2} \sqrt{\frac{a_{1}+\mathrm{i} b_{1}}{p_{1}-p_{1}^{0}}}+O(1)
$$

Since $a_{1}+\mathrm{i} b_{1}$ is a complex number, which is generally non-zero, $\mathrm{d} \lambda / \mathrm{d} p_{1} \rightarrow \infty$ as $p_{1} \rightarrow p_{1}^{0}$.

\subsection{Real eigenvalue $\lambda_{0}$}

Consider the case of a real double eigenvalue $\lambda_{0}$. In this case the eigenvectors $\mathbf{u}_{0}, \mathbf{v}_{0}$ and associated vectors $\mathbf{u}_{1}, \mathbf{v}_{1}$ can be chosen real and, hence, the constants $b_{j}=d_{j}=0, j=1, \ldots, n$, in Eq. (11). Let us fix the increments $\Delta p_{2}, \ldots, \Delta p_{n}$ and consider behaviour of the interacting eigenvalues depending on the increment $\Delta p_{1}$. Then, formula (11) can be written in the form

$$
\lambda=\lambda_{0}+X+\mathrm{i} Y+o(\varepsilon)
$$

where

$$
X+\mathrm{i} Y= \pm \sqrt{a_{1}\left(\Delta p_{1}+\frac{\alpha}{a_{1}}\right)}+c_{1}\left(\Delta p_{1}+\frac{\alpha}{a_{1}}\right)-\frac{c_{1} \alpha}{a_{1}}+\beta,
$$

and $\alpha$ and $\beta$ are small real numbers

$$
\alpha=\sum_{j=2}^{n} a_{j} \Delta p_{j}, \quad \beta=\sum_{j=2}^{n} c_{j} \Delta p_{j}
$$

Real quantities $X$ and $Y$ describe, respectively, the real and imaginary parts of the leading terms in eigenvalue perturbation (11). If $a_{1}\left(\Delta p_{1}+\alpha / a_{1}\right)>0$, then Eq. (15) yields

$$
X= \pm \sqrt{a_{1}\left(\Delta p_{1}+\frac{\alpha}{a_{1}}\right)}+c_{1}\left(\Delta p_{1}+\frac{\alpha}{a_{1}}\right)-\frac{c_{1} \alpha}{a_{1}}+\beta, \quad Y=0 .
$$


If $a_{1}\left(\Delta p_{1}+\alpha / a_{1}\right)<0$, then separating the real and imaginary parts in Eq. (15) gives

$$
\begin{gathered}
X=c_{1}\left(\Delta p_{1}+\frac{\alpha}{a_{1}}\right)-\frac{c_{1} \alpha}{a_{1}}+\beta, \\
Y= \pm \sqrt{-a_{1}\left(\Delta p_{1}+\frac{\alpha}{a_{1}}\right)} .
\end{gathered}
$$

Eliminating $\Delta p_{1}+\alpha / a_{1}$ from Eqs. (18) yields the parabola

$$
X+\frac{c_{1}}{a_{1}} Y^{2}=\beta-\frac{\alpha c_{1}}{a_{1}}
$$

in the $(X, Y)$ plane symmetric with respect to the $X$-axis. Since $\alpha$ and $\beta$ are small numbers depending on $\Delta p_{j}, j=2, \ldots, n$, parabola (19) gives trajectories of $\lambda$ on the complex plane with a change of the parameter $p_{1}$ while the others remain fixed. Note that the constants $a_{1}$ and $c_{1}$ involved in Eq. (19) are taken at the initial point $\mathbf{p}_{0}$ in the parameter space.

First, assume that $a_{1}>0$ and $\Delta p_{2}=\cdots=\Delta p_{n}=0$, which implies $\alpha=\beta=0$. It follows from Eqs. (17)-(19) that with the increase of $\Delta p_{1}$ the eigenvalues come together along parabola (19), merge to $\lambda_{0}$ at $\Delta p_{1}=0$, and then diverge along the real axis in opposite directions. The general picture of strong interaction is shown in Fig. 2, where $\xi=0$ and the arrows indicate motion of the eigenvalues, when $\Delta p_{1}$ increases. The case $a_{1}<0$ implies the change of direction of motion for the eigenvalues.

If $\Delta p_{2}, \ldots, \Delta p_{n}$ are non-zero and fixed, then the constants $\alpha$ and $\beta$ are generally non-zero. This means the shift of parabola (19) along the real axis by $\xi=\beta-\alpha c_{1} / a_{1}$; see Fig. 2. One can see that the double eigenvalue does not disappear. It changes to $\lambda_{0}+\xi+o(\varepsilon)$ and appears at $p_{1}=$ $p_{1}^{0}-\alpha / a_{1}+o(\varepsilon)$.

\subsection{Complex eigenvalue $\lambda_{0}$}

Consider a complex eigenvalue $\lambda_{0}$. In this case the vectors $\mathbf{u}_{0}, \mathbf{u}_{1}, \mathbf{v}_{0}$, and $\mathbf{v}_{1}$ are complex and, hence, the constants $b_{j}$ and $d_{j}$ in expression (11) are generally non-zero. Keeping the lowest order term in Eq. (11) gives

$$
\lambda=\lambda_{0}+X+\mathrm{i} Y+o\left(\varepsilon^{1 / 2}\right)
$$

where

$$
X+\mathrm{i} Y= \pm \sqrt{\sum_{j=1}^{n}\left(a_{j}+\mathrm{i} b_{j}\right) \Delta p_{j}}
$$

$$
a_{1}>0 \quad a_{1}<0
$$
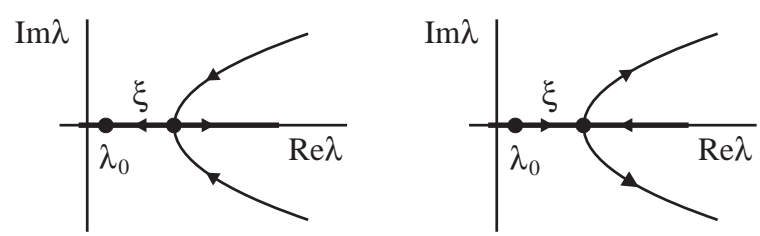

Fig. 2. Strong interaction of eigenvalues (real $\lambda_{0}$ ). 
Taking the square of Eq. (21) and separating real and imaginary parts, one gets the equations

$$
\begin{gathered}
X^{2}-Y^{2}=\sum_{j=1}^{n} a_{j} \Delta p_{j}, \\
2 X Y=\sum_{j=1}^{n} b_{j} \Delta p_{j} .
\end{gathered}
$$

Expressing the increment $\Delta p_{1}$ from one of equations (22) and substituting it into the other equation yields

$$
X^{2}-\frac{2 a_{1}}{b_{1}} X Y-Y^{2}=\gamma
$$

where $\gamma$ is a small real constant:

$$
\gamma=\sum_{j=2}^{n}\left(a_{j}-\frac{a_{1} b_{j}}{b_{1}}\right) \Delta p_{j}
$$

In Eq. (23) it is assumed that $b_{1} \neq 0$, which is the non-degeneracy condition for the complex eigenvalue $\lambda_{0}$. Eq. (23) describes trajectories of the eigenvalues $\lambda$, when only the parameter $\Delta p_{1}$ is changed and the increments $\Delta p_{2}, \ldots, \Delta p_{n}$ are fixed.

If all $\Delta p_{j}=0, j=2, \ldots, n$, or if they are non-zero, but satisfy the equality $\gamma=0$, then Eq. (23) yields two perpendicular lines

$$
\begin{aligned}
& X-\left(\frac{a_{1}}{b_{1}}+\sqrt{1+\frac{a_{1}^{2}}{b_{1}^{2}}}\right) Y=0, \\
& X-\left(\frac{a_{1}}{b_{1}}-\sqrt{1+\frac{a_{1}^{2}}{b_{1}^{2}}}\right) Y=0,
\end{aligned}
$$

which intersect at the origin. Two eigenvalues $\lambda=\lambda_{0}+X+\mathrm{i} Y+o\left(\varepsilon^{1 / 2}\right)$ approach along one of lines (25), merge to $\lambda_{0}$ at $\Delta p_{1}=0$, and then diverge along another line (25), perpendicular to the line of approach; see Fig. 3, where the arrows show motion of $\lambda$ with increasing $\Delta p_{1}$. Strong interaction in the three-dimensional space $\left(p_{1}, \operatorname{Re} \lambda, \operatorname{Im} \lambda\right)$ is shown in Fig. 1 (left) with two identical parabolae lying in perpendicular planes.
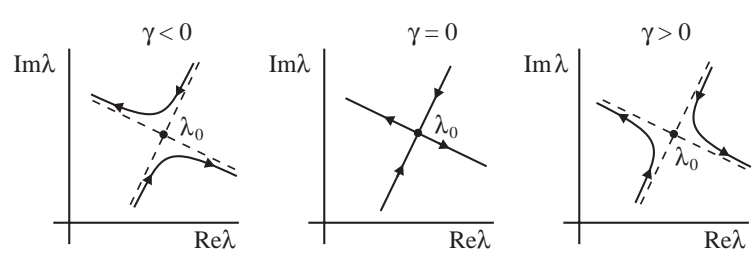

Fig. 3. Strong interaction of eigenvalues (complex $\left.\lambda_{0}\right)$. 
If $\gamma \neq 0$, then Eq. (23) defines two hyperbolae in the (X,Y) plane with asymptotes (25). When $\Delta p_{1}$ increases, two eigenvalues come closer, turn, and diverge; see Fig. 3. When $\gamma$ changes the sign, the quadrants containing hyperbola branches are changed to the adjacent.

Example 1. As an example, stability of vibrations of a rigid panel of infinite span in airflow is considered. It is assumed that the panel is maintained on two elastic supports with the stiffness coefficients $k_{1}$ and $k_{2}$ per unit span. The panel has two degrees of freedom: a vertical displacement $y$ and a rotation angle $\varphi$; see Fig. 4. It is supposed that the aerodynamic lift force $L$ is proportional to the angle of attack $\varphi$, the dynamic pressure of airflow, and the width $b$ of the panel

$$
L=c_{y} \frac{\rho v^{2}}{2} b \varphi .
$$

Here, $c_{y}$ is the aerodynamic coefficient, $\rho$ and $v$ are the density and speed of the flow respectively. It is assumed that $m$ is the mass of the panel per unit surface. Damping forces are not involved in the model.

Small vibrations of the panel in airflow are described by the differential equations [2]

$$
\begin{aligned}
& \ddot{y}+a_{11} y+a_{12} \varphi=0, \\
& \ddot{\varphi}+a_{21} y+a_{22} \varphi=0,
\end{aligned}
$$

where

$$
\begin{gathered}
a_{11}=\frac{k_{1}+k_{2}}{m b}, \quad a_{12}=\frac{k_{1}-k_{2}}{2 m}-c_{y} \frac{\rho v^{2}}{2 m}, \\
a_{21}=\frac{6\left(k_{1}-k_{2}\right)}{m b^{2}}, \quad a_{22}=\frac{3\left(k_{1}+k_{2}\right)}{m b}-3 c_{y} \frac{\rho v^{2}}{2 m b} .
\end{gathered}
$$

It is convenient to introduce the non-dimensional variables

$$
k=\frac{k_{1}-k_{2}}{2\left(k_{1}+k_{2}\right)}, \quad q=\frac{c_{y} \rho v^{2}}{2\left(k_{1}+k_{2}\right)}, \quad \tilde{y}=\frac{y}{b}, \quad \tau=t \sqrt{\frac{k_{1}+k_{2}}{m b}},
$$

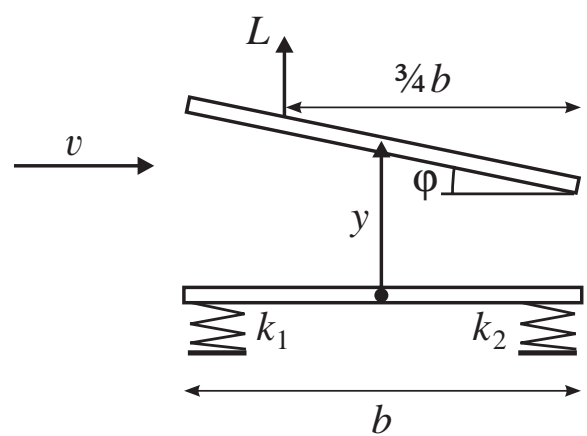

Fig. 4. A panel on elastic supports vibrating in airflow. 
where $k$ is a relative stiffness parameter changing in the interval $-1 / 2 \leqslant k \leqslant 1 / 2$, and $q \geqslant 0$ is a load parameter. Using these variables in Eqs. (27) and separating the time $(\tilde{y}, \varphi)^{\mathrm{T}}=\mathbf{u} \exp (\mathrm{iv} \tau)$, one obtains the eigenvalue problem (1) with

$$
\mathbf{A}=\left(\begin{array}{cc}
1 & k-q \\
12 k & 3-3 q
\end{array}\right), \quad \lambda=v^{2}
$$

The stability problem of motion of the panel depending on two parameters $\mathbf{p}=(q, k)$ has been studied in Ref. [15]. The characteristic equation is

$$
\lambda^{2}+(3 q-4) \lambda+12 k q-3 q-12 k^{2}+3=0 .
$$

Motion of the panel is stable if all eigenvalues $\lambda$ are positive and simple. The stability of the panel can be lost by divergence or by flutter, the boundaries of which are given by $\lambda=0$ or double positive eigenvalues with a single eigenvector respectively. Setting the discriminant of Eq. (31) equal to zero gives

$$
q_{f}=\frac{2}{3}(1+4 k-2 \sqrt{k(k+2)}) .
$$

This is the boundary between flutter and stability regions; see Fig. 5, where $S$ and $F$ denote the stability and flutter regions respectively. It follows from Eq. (32) that the flutter region belongs to the half-plane $k \geqslant 0$. The other branch of the solution, with plus before the square root in Eq. (32), corresponds to the boundary between flutter and divergence regions [15], shown in Fig. 5 by dashed line.

Consider a point $\left(k, q_{f}\right)$ on the flutter boundary (32). At this point, the eigenvalue problem (1) with Eqs. (30) and (32) is solved and the double eigenvalue $\lambda_{0}=2-3 q_{f} / 2$ with the corresponding eigenvectors and associated vectors satisfying normalization conditions (5) are found as

$$
\begin{array}{cl}
\mathbf{u}_{0}=\left(\begin{array}{c}
\frac{2\left(q_{f}-k\right)}{3 q_{f}-2} \\
1
\end{array}\right), \quad \mathbf{u}_{1}=\left(\begin{array}{c}
0 \\
\frac{2}{2-3 q_{f}}
\end{array}\right), \quad \mathbf{v}_{0}=\left(\begin{array}{c}
12 k \\
\frac{2-3 q_{f}}{2}
\end{array}\right), \\
\mathbf{v}_{1}=\left(\begin{array}{c}
\frac{24 k}{3 q_{f}-2} \\
0
\end{array}\right) .
\end{array}
$$

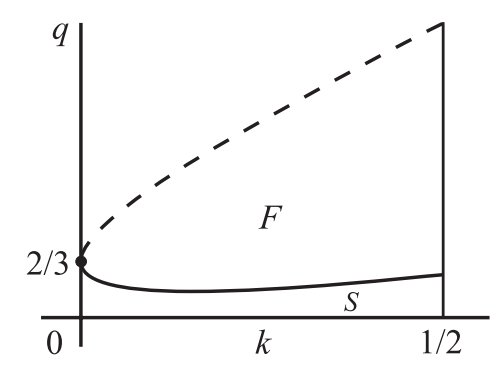

Fig. 5. The stability and flutter regions for the panel vibrating in airflow. 
Then, according to Eqs. (10) the quantities become

$$
\begin{gathered}
a_{1}=-\frac{3}{2}\left(8 k-3 q_{f}+2\right), \quad a_{2}=12\left(2 k-q_{f}\right), \quad c_{1}=-\frac{3}{2}, \quad c_{2}=0, \\
b_{1}=b_{2}=0, \quad d_{1}=d_{2}=0,
\end{gathered}
$$

and the approximate formula (11) for the eigenvalues takes the form

$$
\lambda=\frac{4-3 q_{f}}{2} \pm \sqrt{-\frac{3}{2}\left(8 k-3 q_{f}+2\right) \Delta q+12\left(2 k-q_{f}\right) \Delta k}-\frac{3}{2} \Delta q .
$$

This formula coincides with that of obtained from characteristic equation (31) with first order Taylor expansion of the terms under and out of the square root. The equation of parabola (19) becomes

$$
Y^{2}+\left(8 k-3 q_{f}+2\right) X=12\left(q_{f}-2 k\right) \Delta k .
$$

Due to expressions (32) and (33) the term $a_{1}$ is negative for $0 \leqslant k \leqslant 1 / 2$. This means that for $\Delta k=0$ with the increase of $q$ in the vicinity of the flutter boundary two positive eigenvalues $\lambda$ approach each other, merge to $\lambda_{0}=2-3 q_{f} / 2$, become complex conjugate (flutter) and diverge along parabola (35). If $\Delta k \neq 0$ is small and fixed, then there is a shift of the double eigenvalue by $\xi=-a_{2} c_{1} \Delta k / a_{1}=12\left(q_{f}-2 k\right) \Delta k /\left(8 k-3 q_{f}+2\right)$ and a shift of the parameter $q$ at which the eigenvalue becomes double (flutter boundary)

$$
q_{f}(k+\Delta k) \approx q_{f}(k)-a_{2} \Delta k / a_{1}=q_{f}(k)+\frac{8\left(2 k-q_{f}\right)}{8 k-3 q_{f}+2} \Delta k .
$$

Note that these shifts are negative or positive depending on the sign of $2 k-q_{f}$, which is negative for $0 \leqslant k<2 / \sqrt{3}-1$ and positive for $2 / \sqrt{3}-1<k \leqslant 1 / 2$. At $k=2 / \sqrt{3}-1$ the function $q_{f}(k)$ takes the minimum; see Fig. 5.

\section{Weak interaction}

Consider a double eigenvalue $\lambda_{0}$ of the matrix $\mathbf{A}_{0}$ with two linearly independent eigenvectors. Such an eigenvalue is called semi-simple. Two right eigenvectors $\mathbf{u}_{1}, \mathbf{u}_{2}$ and two left eigenvectors $\mathbf{v}_{1}, \mathbf{v}_{2}$ are determined by the equations

$$
\begin{gathered}
\mathbf{A}_{0} \mathbf{u}_{i}=\lambda_{0} \mathbf{u}_{i}, \quad \mathbf{v}_{i}^{\mathrm{T}} \mathbf{A}_{0}=\lambda_{0} \mathbf{v}_{i}^{\mathrm{T}}, \quad i=1,2, \\
\mathbf{v}_{i}^{\mathrm{T}} \mathbf{u}_{j}=\delta_{i j}, \quad i, j=1,2,
\end{gathered}
$$

where $\delta_{i j}$ is the Kronecker delta. The last equation represents the normalization condition determining left eigenvectors uniquely for given right eigenvectors. Note that any linear combination of right (or left) eigenvectors is a right (or left) eigenvector.

Consider a perturbation of the parameter vector $\mathbf{p}=\mathbf{p}_{0}+\Delta \mathbf{p}$, where $\Delta \mathbf{p}=\varepsilon \mathbf{e}$ with a direction $\mathbf{e}$ in the parameter space $(\|\mathbf{e}\|=1)$ and a small positive perturbation parameter $\varepsilon$. Then, the eigenvalue $\lambda_{0}$ and corresponding eigenvector $\mathbf{u}_{0}$ take increments, which can be given in the form of 
expansions $[9,13]$

$$
\begin{aligned}
& \lambda=\lambda_{0}+\varepsilon \lambda_{1}+\varepsilon^{2} \lambda_{2}+\cdots, \\
& \mathbf{u}=\mathbf{u}_{0}+\varepsilon \mathbf{w}_{1}+\varepsilon^{2} \mathbf{w}_{2}+\cdots
\end{aligned}
$$

By substituting expressions (6) and (38) into eigenvalue problem (1), a chain of equations for the unknowns $\lambda_{1}, \lambda_{2}, \ldots$ and $\mathbf{u}_{0}, \mathbf{w}_{1}, \mathbf{w}_{2}, \ldots$ is obtained. Solution of these equations yields the vector

$$
\mathbf{u}_{0}=\gamma_{1} \mathbf{u}_{1}+\gamma_{2} \mathbf{u}_{2}
$$

where $\gamma_{1}$ and $\gamma_{2}$ are unknown coefficients determined by (see [13])

$$
\left(\begin{array}{cc}
\mathbf{v}_{1}^{\mathrm{T}} \mathbf{A}_{1} \mathbf{u}_{1} & \mathbf{v}_{1}^{\mathrm{T}} \mathbf{A}_{1} \mathbf{u}_{2} \\
\mathbf{v}_{2}^{\mathrm{T}} \mathbf{A}_{1} \mathbf{u}_{1} & \mathbf{v}_{2}^{\mathrm{T}} \mathbf{A}_{1} \mathbf{u}_{2}
\end{array}\right)\left(\begin{array}{l}
\gamma_{1} \\
\gamma_{2}
\end{array}\right)=\lambda_{1}\left(\begin{array}{l}
\gamma_{1} \\
\gamma_{2}
\end{array}\right) .
$$

A non-trivial solution $\gamma_{1}, \gamma_{2}$ of this equation exists if and only if $\lambda_{1}$ is an eigenvalue of the $2 \times 2$ matrix on the left side. Two eigenvalues $\lambda_{1}$ of this matrix and corresponding eigenvectors $\left(\gamma_{1}, \gamma_{2}\right)^{\mathrm{T}}$ determine leading terms in the expansions for two eigenvalues $\lambda$ and corresponding eigenvectors $\mathbf{u}$ (38), which appear due to bifurcation of the double semi-simple eigenvalue $\lambda_{0}$.

Introducing the notation

$$
X+\mathrm{i} Y=\varepsilon \lambda_{1}
$$

where $X$ and $Y$ are, respectively, real and imaginary parts of the term $\varepsilon \lambda_{1}$, expansion for eigenvalue (38) can be written in the form

$$
\lambda=\lambda_{0}+X+\mathrm{i} Y+o(\varepsilon) .
$$

According to relations (7) and (40), $X+\mathrm{i} Y$ is an eigenvalue of the $2 \times 2$ matrix

$$
\sum_{j=1}^{n}\left(\begin{array}{ll}
f_{j}^{11} & f_{j}^{12} \\
f_{j}^{21} & f_{j}^{22}
\end{array}\right) \Delta p_{j}
$$

where

$$
f_{j}^{k l}=\mathbf{v}_{k}^{\mathrm{T}} \frac{\partial \mathbf{A}}{\partial p_{j}} \mathbf{u}_{l},
$$

with the derivatives evaluated at $\mathbf{p}_{0}$. Solving the characteristic equation for matrix (43) gives

$$
X+\mathrm{i} Y=\sum_{j=1}^{n} g_{j} \Delta p_{j} \pm \sqrt{\sum_{j, k=1}^{n} h_{j k} \Delta p_{j} \Delta p_{k}},
$$

where

$$
g_{j}=\left(f_{j}^{11}+f_{j}^{22}\right) / 2, \quad h_{j k}=\left(f_{j}^{11}-f_{j}^{22}\right)\left(f_{k}^{11}-f_{k}^{22}\right) / 4+\left(f_{j}^{12} f_{k}^{21}+f_{j}^{21} f_{k}^{12}\right) / 2 .
$$

Note that $h_{j k}=h_{k j}$ for any $j$ and $k$. Expression (45) determines approximation of eigenvalues (42), when the parameter vector $\Delta \mathbf{p}$ is changing under the assumption that it is small for the absolute value. The coefficients $g_{j}$ and $h_{j k}$ in this expression depend on the left and right eigenvectors, corresponding to the eigenvalue $\lambda_{0}$, and first derivatives of the matrix $\mathbf{A}$ with respect to parameters taken at $\mathbf{p}_{0}$. 


\subsection{Real eigenvalue $\lambda_{0}$}

Consider a real semi-simple eigenvalue $\lambda_{0}$. In this case the eigenvectors $\mathbf{u}_{1}, \mathbf{u}_{2}, \mathbf{v}_{1}, \mathbf{v}_{2}$ can be chosen real and, hence, the coefficients $f_{j}^{k l}, g_{j}$, and $h_{j k}$ in expressions (44) and (46) are real. By expressing the square root from equality (45) and taking the square of the obtained relation, two equations for real and imaginary parts are found as follows:

$$
\begin{gathered}
\left(X-\sum_{j=1}^{n} g_{j} \Delta p_{j}\right)^{2}-Y^{2}=\sum_{j, k=1}^{n} h_{j k} \Delta p_{j} \Delta p_{k}, \\
2\left(X-\sum_{j=1}^{n} g_{j} \Delta p_{j}\right) Y=0 .
\end{gathered}
$$

The second equation requires that $X=\sum_{j=1}^{n} g_{j} \Delta p_{j}$ or $Y=0$. Therefore, two independent systems are obtained:

$$
\left(X-\sum_{j=1}^{n} g_{j} \Delta p_{j}\right)^{2}=\sum_{j, k=1}^{n} h_{j k} \Delta p_{j} \Delta p_{k}, \quad Y=0
$$

and

$$
Y^{2}=-\sum_{j, k=1}^{n} h_{j k} \Delta p_{j} \Delta p_{k}, \quad X=\sum_{j=1}^{n} g_{j} \Delta p_{j}
$$

Consider now the behaviour of eigenvalues depending on the parameter $p_{1}$, when other parameters $p_{2}, \ldots, p_{n}$ are fixed. First, let us put the increments $\Delta p_{2}=\cdots=\Delta p_{n}=0$. Then, Eqs. (48) and (49) take the form

$$
\begin{gathered}
\left(X-g_{1} \Delta p_{1}\right)^{2}=h_{11} \Delta p_{1}^{2}, \quad Y=0, \\
Y^{2}=-h_{11} \Delta p_{1}^{2}, \quad X=g_{1} \Delta p_{1} .
\end{gathered}
$$

Upon assuming that $h_{11} \neq 0$ (the non-degenerate case), only one of systems (50) and (51) has nonzero solutions. If $h_{11}>0$, then system (51) has only the zero solution $X=Y=\Delta p_{1}=0$, and system (50) yields

$$
X=g_{1} \Delta p_{1} \pm \sqrt{h_{11}} \Delta p_{1}, \quad Y=0 .
$$

Expression (52) describe two real eigenvalues (42), which cross each other at the point $\lambda_{0}$ on the complex plane as $\Delta p_{1}$ changes from negative to positive values; see Fig. $6\left(r^{\prime}\right)$, where the arrows show motion of the eigenvalues with an increment of $\Delta p_{1}$. If $h_{11}<0$, then system (50) has only the zero solution, and system (51) yields

$$
X=g_{1} \Delta p_{1}, \quad Y= \pm \sqrt{-h_{11}} \Delta p_{1} .
$$

These formulae describe two complex conjugate eigenvalues crossing at the point $\lambda_{0}$ on the real axis with a change of $\Delta p_{1}$; see Fig. $6\left(r^{\prime \prime}\right)$. 


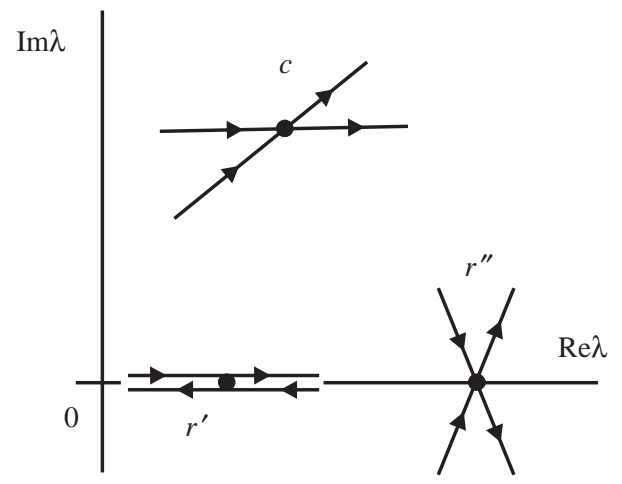

Fig. 6. Weak interaction of eigenvalues for $\Delta p_{2}=\cdots=\Delta p_{n}=0$.

From expressions (52) and (53) one can see that the weak interaction occurs at the same plane in the three-dimensional space $\left(p_{1}, \operatorname{Re} \lambda, \operatorname{Im} \lambda\right)$, and the speed of interaction $\mathrm{d} \lambda / \mathrm{d} p_{1}$ remains finite, Fig. 1 (right).

Now, consider the case, when the increments $\Delta p_{2}, \ldots, \Delta p_{n}$ are small and fixed. Then, upon using the notation

$$
\sum_{j=1}^{n} g_{j} \Delta p_{j}=g_{1} \Delta p_{1}+\sigma, \quad \sum_{j, k=1}^{n} h_{j k} \Delta p_{j} \Delta p_{k}=h_{11}\left(\Delta p_{1}-\delta\right)^{2}+\psi,
$$

where $\sigma, \delta$, and $\psi$ are small real constants depending on $\Delta p_{2}, \ldots, \Delta p_{n}$,

$$
\sigma=\sum_{j=2}^{n} g_{j} \Delta p_{j}, \quad \delta=-\sum_{j=2}^{n} \frac{h_{1 j}}{h_{11}} \Delta p_{j}, \quad \psi=\sum_{j, k=2}^{n} h_{j k} \Delta p_{j} \Delta p_{k}-h_{11} \delta^{2},
$$

Eqs. (48) and (49) take the form

$$
\begin{gathered}
\left(X-\sigma-g_{1} \Delta p_{1}\right)^{2}-h_{11}\left(\Delta p_{1}-\delta\right)^{2}=\psi, \quad Y=0, \\
Y^{2}+h_{11}\left(\Delta p_{1}-\delta\right)^{2}=-\psi, \quad X=\sigma+g_{1} \Delta p_{1} .
\end{gathered}
$$

Solutions of systems (56) and (57) depend qualitatively on the signs of the constants $h_{11}$ and $\psi$. Under the non-degeneracy conditions $h_{11} \neq 0$ and $\psi \neq 0$ there are four possibilities.

Case $r_{+}^{\prime}\left(h_{11}>0, \psi>0\right)$ : System (56) determines two hyperbolae in the $\left(\Delta p_{1}, X\right)$ plane; system (57) has no solutions; see Fig. 7. Two simple real eigenvalues approach, and then diverge as $\Delta p_{1}$ is changed; a double eigenvalue does not appear; see Fig. 8.

Case $r_{-}^{\prime}\left(h_{11}>0, \psi<0\right)$ : System (56) determines two hyperbolae in the $\left(\Delta p_{1}, X\right)$ plane; system (57) defines an ellipse in the $\left(\Delta p_{1}, Y\right)$ plane; see Fig. 7. The hyperbolae and ellipse have two common points

$$
\Delta p_{1}^{ \pm}=\delta \pm \sqrt{-\psi / h_{11}}, \quad X^{ \pm}=\sigma+g_{1} \Delta p_{1}^{ \pm}, \quad Y^{ \pm}=0 .
$$

With increasing $\Delta p_{1}$ two simple real eigenvalues approach, interact strongly at $\Delta p_{1}^{-}=$ $\delta-\sqrt{-\psi / h_{11}}$, become complex conjugate, interact strongly again at $\Delta p_{1}^{+}=\delta+\sqrt{-\psi / h_{11}}$, and then diverge along the real axis; see Fig. 8. By eliminating $\Delta p_{1}$ from Eq. (57), an ellipse on the 

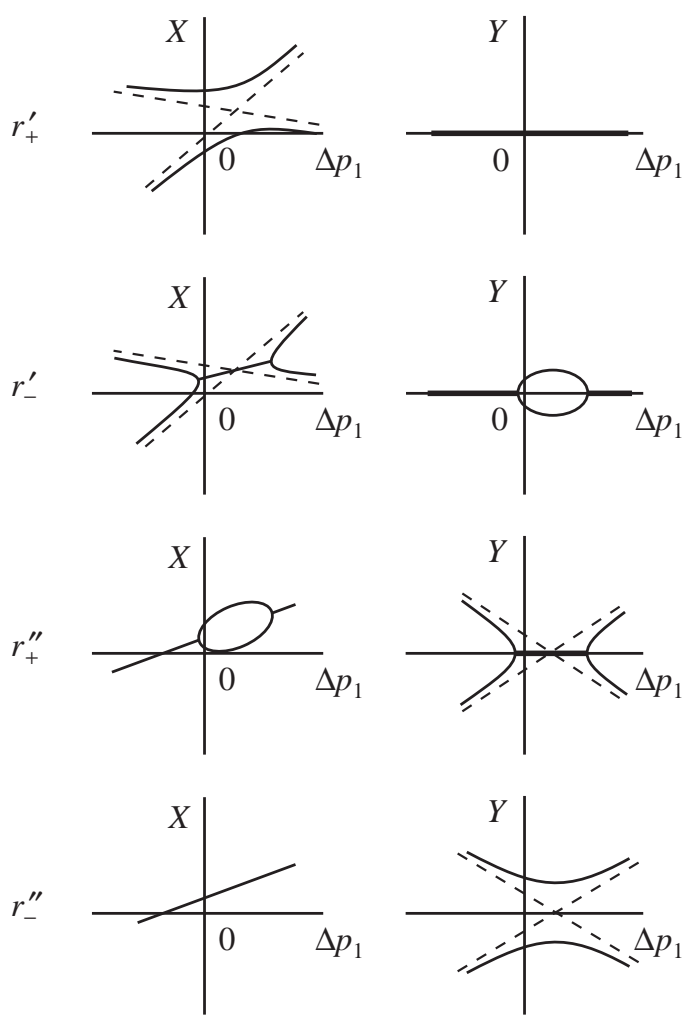

Fig. 7. Weak interaction of eigenvalues for small $\Delta p_{2}, \ldots, \Delta p_{n}$.

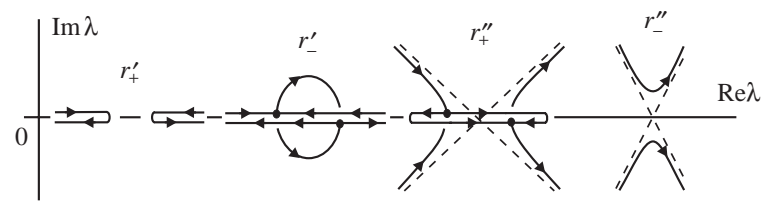

Fig. 8. Weak interaction of eigenvalues on the complex plane for small $\Delta p_{2}, \ldots, \Delta p_{n}$.

complex plane is obtained as

$$
Y^{2}+h_{11}\left(\frac{X-\sigma-g_{1} \delta}{g_{1}}\right)^{2}=-\psi
$$

see Fig. 8.

If the eigenvalues are plotted in the $\left(p_{1}, \operatorname{Re} \lambda, \operatorname{Im} \lambda\right)$ space, one observes a small elliptic bubble appearing from the point $\left(p_{1}^{0}, \lambda_{0}, 0\right)$; see Fig. 9 . This bubble is placed in the plane perpendicular to the plane of original interaction.

At points (58) the double real eigenvalues

$$
\lambda^{ \pm}=\lambda_{0}+X^{ \pm}+o(\varepsilon)
$$



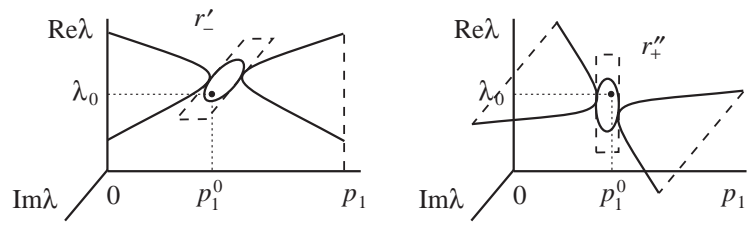

Fig. 9. Weak interaction of eigenvalues in the $\left(p_{1}, \operatorname{Re} \lambda, \operatorname{Im} \lambda\right)$ space for small $\Delta p_{2}, \ldots, \Delta p_{n}$ in the cases $r_{-}^{\prime}$ and $r_{+}^{\prime \prime}$.

appear. It is easy to show that each of these eigenvalues has a single eigenvector. Indeed, if eigenvalues (60) were semi-simple, then $X^{ \pm}$have to be semi-simple eigenvalues of $2 \times 2$ matrix (43) at $\Delta p_{1}=\Delta p_{1}^{ \pm}$. Hence, matrix (43) becomes

$$
\left(\begin{array}{cc}
X_{ \pm}^{ \pm}+f_{1}^{11}\left(\Delta p_{1}-\Delta p_{1}^{ \pm}\right) & f_{1}^{12}\left(\Delta p_{1}-\Delta p_{1}^{ \pm}\right) \\
f_{1}^{21}\left(\Delta p_{1}-\Delta p_{1}^{ \pm}\right) & X^{ \pm}+f_{1}^{22}\left(\Delta p_{1}-\Delta p_{1}^{ \pm}\right)
\end{array}\right) .
$$

Using this matrix with expressions (46) and (54), gives

$$
\begin{aligned}
h_{11}\left(\Delta p_{1}-\delta\right)^{2}+\psi & =\sum_{j, k=1}^{n} h_{i j} \Delta p_{j} \Delta p_{k} \\
& =\left(\left(f_{1}^{11}-f_{1}^{22}\right)^{2} / 4+f_{1}^{12} f_{1}^{21}\right)\left(\Delta p_{1}-\Delta p_{1}^{ \pm}\right)^{2}=h_{11}\left(\Delta p_{1}-\Delta p_{1}^{ \pm}\right)^{2}
\end{aligned}
$$

and, hence, $\psi=0$. But this is a contradiction to the assumption that $\psi<0$. Therefore, two interactions at points (58) are strong and follow the scenarios described in the previous section.

Case $r_{+}^{\prime \prime}\left(h_{11}<0, \psi>0\right)$ : System (56) determines an ellipse in the $\left(\Delta p_{1}, X\right)$ plane; system $(57)$ defines two hyperbolae in the $\left(\Delta p_{1}, Y\right)$ plane; see Fig. 7. The hyperbolae and ellipse have two common points (58), where double real eigenvalues (60) appear and cause strong interactions of eigenvalues. Therefore, with a monotonous change of $\Delta p_{1}$ two complex conjugate eigenvalues approach, interact strongly at $\Delta p_{1^{-}}=\delta-\sqrt{-\psi / h_{11}}$, become real, interact again at $\Delta p_{1^{+}}=$ $\delta+\sqrt{-\psi / h_{11}}$, then become complex conjugate and diverge; see Fig. 8. For this case Eq. (59) gives hyperbolae on the complex plane. The behaviour of eigenvalues in the three-dimensional space $\left(p_{1}, \operatorname{Re} \lambda, \operatorname{Im} \lambda\right)$ is shown in Fig. 9, where one can see a small elliptic bubble appearing in the plane $\operatorname{Im} \lambda=0$ perpendicular to the plane of original interaction.

Case $r_{-}^{\prime \prime}\left(h_{11}<0, \psi<0\right)$ : System (56) has no solutions; system (57) determines two hyperbolae in the $\left(\Delta p_{1}, Y\right)$ plane symmetric with respect to the $\Delta p_{1}$ axis; see Fig. 7 . Two complex conjugate eigenvalues approach, and then diverge with an increment of $\Delta p_{1}$; a double eigenvalue does not appear; see Fig. 8. Note that hyperbolae (59) change the vertical angles, where they appear, compared to the case $r_{+}^{\prime \prime}$.

Variations of the parameters $\Delta p_{2}, \ldots, \Delta p_{n}$ change a picture of weak interaction in two ways: either the double semi-simple real eigenvalue disappear and simple eigenvalues move along hyperbolae as $\Delta p_{1}$ changes, or the double semi-simple eigenvalue splits in two double eigenvalues with single eigenvectors, which leads to a couple of successive strong interactions with the appearance of a small bubble in the $\left(p_{1}, \operatorname{Re} \lambda, \operatorname{Im} \lambda\right)$ space. 


\subsection{Complex eigenvalue $\lambda_{0}$}

Finally, consider the case when a double semi-simple eigenvalue $\lambda_{0}$ is complex. In this case the eigenvalues $\mathbf{u}_{1}, \mathbf{u}_{2}, \mathbf{v}_{1}, \mathbf{v}_{2}$ and, hence, the coefficients $f_{j}^{k l}, g_{j}$, and $h_{j k}$ are complex. If $\Delta p_{2}=\cdots=$ $\Delta p_{n}=0$, then expression (45) yields

$$
X+\mathrm{i} Y=\left(g_{1} \pm \sqrt{h_{11}}\right) \Delta p_{1},
$$

where $g_{1}$ and $h_{11}$ are complex numbers. With a change of $\Delta p_{1}$ two eigenvalues (42) cross each other at the point $\lambda_{0}$ on the complex plane; see Fig. $6(c)$.

Upon assuming that the increments $\Delta p_{2}, \ldots, \Delta p_{n}$ are small and fixed, Eq. (45) yields

$$
X+\mathrm{i} Y=\sigma+g_{1} \Delta p_{1} \pm \sqrt{h_{11}} \sqrt{\left(\Delta p_{1}-\delta\right)^{2}+\psi / h_{11}}
$$

where $\sigma, \delta$, and $\psi$ are small complex numbers defined by expressions (55). Upon assuming that the second term under the square root in Eq. (64) is much smaller than the first term, the following formula can be deduced:

$$
\begin{aligned}
X+\mathrm{i} Y & \approx \sigma+g_{1} \delta+g_{1}\left(\Delta p_{1}-\delta\right) \pm \sqrt{h_{11}}\left(\left(\Delta p_{1}-\delta\right)+\frac{\psi}{2 h_{11}\left(\Delta p_{1}-\delta\right)}\right) \\
& =\sigma+g_{1} \delta+\left(g_{1} \pm \sqrt{h_{11}}\right)\left(\Delta p_{1}-\delta\right)+o\left(\Delta p_{1}-\delta\right)
\end{aligned}
$$

showing that the main directions of eigenvalues on the complex plane before and after the weak interaction remain the same as for unperturbed case (63).

The expression under the square root in Eq. (64)

$$
z=\left(\Delta p_{1}-\delta\right)^{2}+\psi / h_{11},
$$

defines a parabola in the complex plane with a change of $\Delta p_{1}$; see Fig. 10 (in the case $\operatorname{Im} \delta=0$ the parabola degenerates to a ray). Computing points $z_{1}$ and $z_{2}$ of the parabola belonging to the imaginary axis, which is perpendicular to the axis of the parabola, results in

$$
\eta=z_{1} z_{2}=4(\operatorname{Im} \delta)^{4}-4(\operatorname{Im} \delta)^{2} \operatorname{Re} \frac{\psi}{h_{11}}-\left(\operatorname{Im} \frac{\psi}{h_{11}}\right)^{2} \in \mathbb{R} .
$$

Assume that $\eta \neq 0$, which is a non-degenerate case. This means that $z \neq 0$ for all $\Delta p_{1}$ and, hence, two values of $X+\mathrm{i} Y$ given by expression (64) are different. As a result, eigenvalues (42) are different and the double eigenvalue disappears.

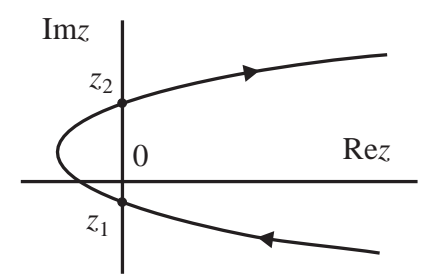

Fig. 10. Image of the function $z\left(\Delta p_{1}\right)$ with monotonic change of $\Delta p_{1}$. 
If $\eta>0$, then two purely imaginary points $z_{1}$ and $z_{2}$ lie at different sides of the origin, i.e., the origin belongs to the interior of the parabola. In this case $z$ makes a turn around the origin as $\Delta p_{1}$ changes. This means that eigenvalues (42) approach, and then diverge without a change of direction as shown in Fig. $11\left(c_{+}\right)$. If $\eta<0$, then the origin lies outside the parabola (this condition remains valid, when the parabola does not intersect the imaginary axis). As a result, eigenvalues (42) approach, and then diverge with a change of direction as shown in Fig. $11\left(c_{-}\right)$.

Variations of the parameters $\Delta p_{2}, \ldots, \Delta p_{n}$ destroy a double semi-simple complex eigenvalue. A picture of weak interaction can change in two ways: either eigenvalues follow the same directions after passing the neighbourhood of $\lambda_{0}$, or the eigenvalues interchange their directions. Behaviour of the eigenvalues in the neighbourhood of $\lambda_{0}$ can be rather complicated due to the square root of complex expression in formula (64).

Example 2. Consider a linear conservative system

$$
\mathbf{M} \ddot{\mathbf{x}}+\mathbf{C x}=0,
$$

where $\mathbf{x} \in \mathbb{R}^{m}$ is a vector of generalized coordinates; $\mathbf{M}$ and $\mathbf{C}$ are symmetric positive definite real matrices of size $m \times m$ smoothly depending on a vector of two real parameters $\mathbf{p}=\left(p_{1}, p_{2}\right)$. Taking a solution of this system in the form $\mathbf{x}=\mathbf{u} \exp (\mathrm{i} \omega t)$, the following eigenvalue problem is obtained,

$$
\mathbf{C u}=\omega^{2} \mathbf{M u},
$$

where $\omega \in \mathbb{R}$ is a frequency and $\mathbf{u}$ is a mode of vibrations. Upon denoting

$$
\mathbf{A}=\mathbf{M}^{-1} \mathbf{C}, \quad \lambda=\omega^{2},
$$

Eq. (69) can be written in standard form (1).

Consider now a point $\mathbf{p}_{0}$ in the parameter space, where the matrix $\mathbf{A}_{0}=\mathbf{M}_{0}^{-1} \mathbf{C}_{0}$ has a double eigenvalue $\lambda_{0}=\omega_{0}^{2}$. Since the matrices $\mathbf{M}_{0}$ and $\mathbf{C}_{0}$ are symmetric, the multiple eigenvalue $\lambda_{0}$ is always semi-simple. Let $\mathbf{u}_{1}$ and $\mathbf{u}_{2}$ be right eigenvectors (modes) corresponding to the eigenvalue $\lambda_{0}$. It can be shown that left eigenvectors are

$$
\mathbf{v}_{1}=\mathbf{M}_{0} \mathbf{u}_{1}, \quad \mathbf{v}_{2}=\mathbf{M}_{0} \mathbf{u}_{2} .
$$

Normalization conditions (37) take the form

$$
\mathbf{u}_{1}^{\mathrm{T}} \mathbf{M}_{0} \mathbf{u}_{1}=\mathbf{u}_{2}^{\mathrm{T}} \mathbf{M}_{0} \mathbf{u}_{2}=1, \quad \mathbf{u}_{1}^{\mathrm{T}} \mathbf{M}_{0} \mathbf{u}_{2}=\mathbf{u}_{2}^{\mathrm{T}} \mathbf{M}_{0} \mathbf{u}_{1}=0 .
$$

Using expressions (70) and (71) in formula (44) gives

$$
f_{j}^{k l}=\mathbf{u}_{k}^{\mathrm{T}}\left(\frac{\partial \mathbf{C}}{\partial p_{j}}-\omega_{0}^{2} \frac{\partial \mathbf{M}}{\partial p_{j}}\right) \mathbf{u}_{l},
$$
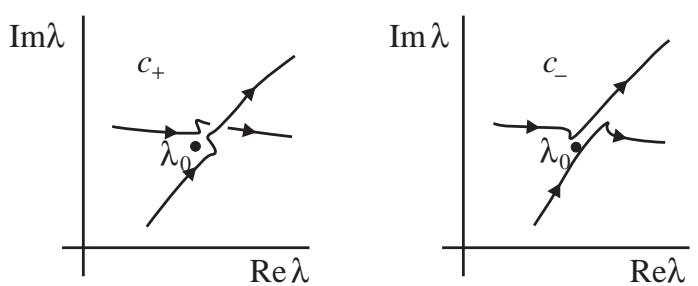

Fig. 11. Weak interaction of eigenvalues for small $\Delta p_{2}, \ldots, \Delta p_{n}$ (complex $\lambda_{0}$ ). 
where $f_{j}^{12}=f_{j}^{21}$ due to the symmetry of the matrices $\mathbf{M}$ and $\mathbf{C}$. With the use of relations (46), one obtains

$$
g_{1}=\left(f_{1}^{11}+f_{1}^{22}\right) / 2, \quad h_{11}=\left(f_{1}^{11}-f_{1}^{22}\right)^{2} / 4+\left(f_{1}^{12}\right)^{2} \geqslant 0 .
$$

Upon assuming that $h_{11} \neq 0$, the bifurcation of the double eigenvalue $\lambda_{0}$ is given by expression (52) for the case $\Delta p_{2}=0$. This bifurcation is of the type $r^{\prime}$, see Fig. 6 , where $\lambda_{0}$ splits into two real eigenvalues for small increments $\Delta p_{1}$. This agrees with the general theory, which says that all frequencies of the conservative system under consideration are real. If $\Delta p_{2}$ is non-zero and small, then expression (55) gives

$$
\begin{gathered}
\sigma=\left(f_{2}^{11}+f_{2}^{22}\right) \Delta p_{2} / 2, \\
\delta=-\left(\left(f_{1}^{11}-f_{1}^{22}\right)\left(f_{2}^{11}-f_{2}^{22}\right) / 4+f_{1}^{12} f_{2}^{12}\right) \Delta p_{2} / h_{11}, \\
\psi=\left(\left(f_{1}^{11}-f_{1}^{22}\right) f_{2}^{12}-\left(f_{2}^{11}-f_{2}^{22}\right) f_{1}^{12}\right)^{2}\left(\Delta p_{2}\right)^{2} /\left(4 h_{11}\right) \geqslant 0 .
\end{gathered}
$$

Hence, behaviour of the eigenvalues with a change of $\Delta p_{1}$ is described by two hyperbolae (56); see Figs. 7 and $8\left(r_{+}^{\prime}\right)$. Two real eigenvalues approach, turn at some distance from each other, and diverge with a monotonic change of $\Delta p_{1}$. The frequencies

$$
\omega=\sqrt{\lambda}=\sqrt{\omega_{0}^{2}+X+\mathrm{i} Y+o(\varepsilon)}=\omega_{0}+\frac{X+\mathrm{i} Y}{2 \omega_{0}}+o(\varepsilon),
$$

have the same type of behaviour in the neighbourhood of $\omega_{0}$. A small perturbation of the second parameter $\Delta p_{2}$ destroys a picture of weak interaction in such a way that the double frequency disappears. This agrees with the results of singularity theory [21].

\section{Conclusion}

A new theory of interaction of eigenvalues in multi-parameter problems has been presented. This theory describes behaviour of eigenvalues with a change of parameters based on the information at the initial point, where the eigenvalues coincide. This information includes determination of the eigenvectors and associated vectors and first order derivatives of the system matrix with respect to parameters. The presented theory of interaction of eigenvalues in multiparameter problems has a very broad field of applications because any physical system contains parameters. The study of behaviour of eigenvalues in vibrational systems is especially important for dynamic stability problems, since even a small change of parameters can lead to instability and catastrophic response of the system.

Interaction of two eigenvalues, which is obviously the most important case frequently taking place in vibrational problems, has been studied. The study of eigenvalues of higher multiplicity as well as investigation of some degenerate cases can be done using the methods developed in the present paper. 


\section{References}

[1] V.V. Bolotin, Nonconservative Problems of the Theory of Elastic Stability, Pergamon, New York, 1963.

[2] Ya.G. Panovko, I.I. Gubanova, Stability and Oscillations of Elastic Systems, Consultants Bureau, New York, 1965.

[3] H. Ziegler, Principles of Structural Stability, Blaisdell, Waltham, MA, 1968.

[4] K. Huseyin, Vibrations and Stability of Multiple Parameter Systems, Sijthoff \& Nordhoff, Alphen aan den Rijn, 1978.

[5] H. Leipholz, Stability Theory, B.G. Teubner, Stuttgart, 1987.

[6] J.M.T. Thompson, Instabilities and Catastrophes in Science and Engineering, Wiley, London, 1982.

[7] J.J. Thomsen, Vibrations and Stability. Order and Chaos, McGraw-Hill, London, 1997.

[8] M.P. Paidoussis, Fluid-Structure Interactions: Slender Structures and Axial Flow, Vol. 1, Academic Press, London, 1998.

[9] M.I. Vishik, L.A. Lyusternik, The solution of some perturbation problems for matrices and selfadjoint or nonselfadjoint differential equations, Russian Mathematical Surveys 15 (1960) 1-74.

[10] V.B. Lidskii, Perturbation theory of non-conjugate operators, USSR Computational Mathematics and Mathematical Physics 6 (1) (1966) 73-85.

[11] J. Moro, J.V. Burke, M.L. Overton, On the Lidskii-Vishik-Lyusternik perturbation theory for eigenvalues of matrices with arbitrary Jordan structure, SIAM Journal on Matrix Analysis and Applications 18 (4) (1997) $793-817$.

[12] A.P. Seyranian, Sensitivity analysis of eigenvalues and development of instability, Strojnicky Casopis 42 (3) (1991) 193-208.

[13] A.P. Seyranian, Sensitivity analysis of multiple eigenvalues, Mechanics of Structures and Machines 21 (2) (1993) 261-284.

[14] A.A. Mailybaev, A.P. Seyranian, On singularities of a boundary of the stability domain, SIAM Journal on Matrix Analysis and Applications 21 (1) (1999) 106-128.

[15] A.P. Seyranian, O.N. Kirillov, Bifurcation diagrams and stability boundaries of circulatory systems, Theoretical and Applied Mechanics 26 (2001) 135-168.

[16] A.P. Seyranian, Collisions of eigenvalues in linear oscillatory systems, Journal of Applied Mathematics and Mechanics 58 (5) (1994) 805-813.

[17] A.P. Seyranian, P. Pedersen, On two effects in fluid/structure interaction theory, in: P.W. Bearman (Ed.), Flow Induced Vibration, A.A. Balkema, Rotterdam, 1995, pp. 565-576.

[18] O.N. Kirillov, A.P. Seyranian, Overlapping of frequency curves in nonconservative systems, Doklady Physics 46 (3) (2001) 184-189.

[19] A.A. Mailybaev, A.P. Seyranian, The stability domains of Hamiltonian systems, Journal of Applied Mathematics and Mechanics 63 (4) (1999) 545-555.

[20] A.P. Seyranian, W. Kliem, Bifurcations of eigenvalues of gyroscopic systems with parameters near stability boundaries, ASME Journal of Applied Mechanics 68 (2) (2001) 199-205.

[21] V.I. Arnold, Mathematical Methods of Classical Mechanics, Springer, New York, 1978. 